\title{
Analysis of the work of air traffic controllers of the approach control area (APP) of Porto Alegre, Brazil
}

\author{
C. V. Vargas ${ }^{a^{*}}$, L. B. de M. Guimarães ${ }^{a}$ and A. M. O. Sant'Anna ${ }^{\text {b }}$ \\ ${ }^{a}$ Graduate Program in Industrial Engineering, Federal University of Rio Grande do Sul, Av. Osvaldo Aranha 99 \\ $5^{\circ}$ andar, Porto Alegre, RS, 90035-190,Brazil,E-mail: liabmg@gmail.com \\ ${ }^{b}$ Industrial and Systems Graduate Program, Pontificie Universidade Catolica do Parana, Rua Imaculada \\ Conceicão, 1155, Prado Velho, Curitiba,PR, 80215-901,Brazil, E-mail: angeloms@gmail.com Tel: +55 41 \\ 32711332
}

\begin{abstract}
This article presents a study on the activities of the air traffic controllers of the Approach Control Area (APP) of Porto Alegre, Brazil, in different real scenarios. Based on interviews, questionnaires and the analysis of film of real scenes, the following were identified and analyzed: i) the perceptions of risk and complexity of the different air traffic scenes observed; ii) the cognitive factors (knowledge, strategy and attention dynamics) involved in the task and iii) the perception of the controller's workload. The results showed that the task complexity depends on the weather conditions, the number and type of aircraft in observation and that the controllers perceive the scenes in a similar way irrespective of their time in the profession and the type of control (radar or coordination). Attention is the cognitive factor with the greatest impact on the work and mental demand has the greatest impact on workload followed by time demand. The literature on the controllers work in Brazil is scarce and, therefore, this study aimed to contribute to the understanding of the work in one APP in order to promote future changes in the very problematic Brazilian air traffic system.
\end{abstract}

Keywords: air traffic control, complexity, safety, workload.

\section{Introduction}

Brazilian air traffic has been increasing year by year, which has led to there being, in some points of the national territory, an excess of aircraft traffic, with the consequent congestion of airports and flight paths. There are 2,700 air traffic controllers in Brazil, mostly military personnel and a further 800 would be needed for the monitoring of aircraft to be performed safely [27]. The consequence of this is that these professionals are overloaded and control more aircrafts than the number laid down in regulations. According to aviation legislation ICA 100-30 [4], the maximum number of aircraft controlled by a controller is determined in accordance with direct and inverse func-

* Corresponding author. Email: cleytonfisio@yahoo.com.br tel: +55 (51) 3308-3948 fax: +55 (51) 3308-4007 tions. The direct functions include the factors of the availability of the controller and the average time that the aircraft remain in the sector. The inverse functions are the factors of the number of communications per each aircraft in the sector and the average duration of each message. These values are gathered taking into consideration the movement of aircraft at the moments when there is the largest number of aircraft. Thus, there are variations between the different control bodies and the locality considered. In Porto Alegre, Rio Grande do Sul, for example, there are up to ten aircraft in operations using radar equipment and six aircraft without radar equipment for each controller provided that the controller has an assistant.

In Brazil, the option was taken, in the 1970s, to create a single system to control both civil and military aviation, contrary to what happens in other countries. The air traffic control (ATC) is commanded by a set of four tracking units that are integrated so as to 
cover the whole national territory. Each regional unit of the system bears the name of Integrated Centre of Air Defense and Airspace Control (CINDACTA), each one having an Area Control Centre (ACC) and a Centre for Military Operations (COPM) responsible for the management of civil and military air traffic control, respectively.

The Brazilian ATC system is fragile. It is subject to problems that range from the lack of maintaining equipment to the recent strikes of the operators, who are insufficient in number. Fifteen years ago, the country had 3,200 controllers. Since then, the fleet of aircraft and air traffic have nearly doubled, but the number of controllers has gone on decreasing. Another problem is the decline of the professionals qualification [12]. It is estimated that today, only $10 \%$ of controllers speak fluent English, the official language used in contacts with foreign pilots [12]. In 2006, the Brazilian ATC system underwent its greatest crisis, which generated aircraft delay, radar crash and airports closing, what has led to the investigation of several possible causes: if the procedures were following the rules (e.g., longitudinal and vertical separation between aircraft, flight plan, phraseology), the quality of communication between the different air traffic control centres, the formal training and further skills-acquisitions of the air traffic controller, the technology available, the workload that the professionals in the industry are exposed to, the management and constraints of the system and so forth [1].

Despite the problems that the system faces, the Brazilian airspace system is under military control, what explains the few published studies on the topic focusing on the technology, the workroom design $[24,25]$ and the shift work in ATC [24]. Such a scarce literature does not help to promote a better and safer system for both workers and travelers.

In order to contribute to the literature of the Brazilian ATC system, this article presents a brief literature review on ATC and a study [26] on the activities of air traffic controllers at the Approach Control Area (APP) from Porto Alegre (APP-PA).

\section{Characterization of the air traffic control (ATC) system}

The ATC system was described as human performance interfaces in which the air traffic controllers interact with other human performance factors including other controllers, software, hardware, envi- ronment, and organization, the later playing a more significant role than individual differences or peer influences on how the controllers interact with the ATC system [5].

ATC was also described as a complex system, which is characterized, among other factors, by the dynamism, the complexity of the factors involved, making decisions under time-pressure (i.e. avoid obstacles, assess dangers, select the data they need to assess and manage different forms of the system behavior) multiple conflicts of goals and pressure to obtain results [16]. Because of its dynamic character, the state of the system changes independently and, as the result of someone taking an action, several interdependent decisions in real time is required. Events happen quickly, are often complex and consist of several components that require cognitive effort and skill from the controller who has to review and make a decision under a lot of time pressure, and quickly change his/her strategy of action in order to adjust to each situation [2].

\section{The task and the taskload of the air traffic controller}

The task of an air traffic controller can be grouped into three main categories [20]: 1) communication that includes actions or utterances initiated by the tactical controller, such as accept, navigate, hand off, query and negotiate; 2) flight progress activities that deal with the paper strips including manipulation and remove; and 3) radar activities such as tidy the radar screen.

Air traffic controllers are subject to the momentby-moment situation of air traffic meaning that, as per the variation in traffic, the need increases for the rapid "resolution" of "conflicts". The variation of the flow of aircraft in the same airspace leads to the controller having to work with a greater number of aircraft close to each other and, in some cases, having to deal, at the same time, with a larger than expected number of aircrafts [10]. In performing their activities, controllers have to deal with different scenarios, of different levels of complexity, as many or few variables may be involved. They have to coordinate their decisions, which must be based on knowledge, and act in a way that the system under control is efficient and safe. It should be also emphasized that weather conditions add to this complexity [19] because it is the factor most subject to variations (wind speed, rain, clouds) and when there are unexpected 
changes, this can directly bear on the safety of managing the traffic.

The three key elements in the work of ATC are efficiency, safety buffer and task load [14]. It is considered efficient when it guarantees separation standards (typically 3 or 5 miles horizontally and 1000 feet vertically [6] with smooth flight paths, a minimum of additional changes in direction and speed as well as responding to the requests from pilots as far as possible to save time and fuel [19]. Safety is minimally achieved when the risk of most characteristic incidents in the air traffic system is minimized [1]: 1) a near collision of aircraft; 2) a grave difficulty caused by failure to carry out operational procedures of cabin; 3) irregularity or failures of operational procedures of airspace control. In accordance with the ISO 10075 norms $[17,18]$, task load or demand are the regular terms normally described by task analysis. It refers to external factors, which result in different amounts of workload on the side of the operators, depending on their skills, ways of working and their state. The concept of workload refers to strain (i.e. stress response), which is elicited by the task (load), which corresponds to stressors (i.e. stress-eliciting situations).

Studies on the workload of the ATC controllers evaluated the psychological and physiological effects of the work $[8,32]$, the dynamic aspects of the relationship between time and actions [9,29] and how increasing task load (i.e. increasing traffic complexity) will increase workload and, consequently will increase the probability of errors and will reduce safety buffers $[3,23,28]$. Therefore, the analysis of situational demands (task load) is a prerequisite of determining the factors that influence workload in ATC. The task load in air traffic control has to be limited or at least monitored to keep safety on a high level and to keep the operators' workload well manageable [19]. Task load does not only determine workload but also has an impact on safety buffers and efficiency. If task load becomes too high, the air traffic controller may decide to either reduce safety buffers to keep efficiency high or keep the actual safety buffer level, thus reducing efficiency. The limits of the controller task load are determined by the capacity limits of a given sector. Safety buffers are set by the controller in accordance with air traffic regulations to keep system safety in critical situations: they are additional risk-reducing factors, which are introduced in the system or inherent in the system to increase safety beyond tolerable risk levels because they allow controllers to cope with errors, mishaps or deviations from normal procedures and help to prevent critical incidents and accidents.

Kuk, Arnold and Ritter [20] analyzed how the operations of an air traffic controller change under light and heavy traffic workload addressing the underlying structural and temporal relationships among various discrete activities in ATC operations. They found that the effect of time allocation is a greater determinant than other variables examined in the study and is more prominent in heavy rather than in light workload conditions. This generally reflects that time has to be more efficiently used and distributed among activities for combating the increasing task demands when workload increases. On the other hand, under the light workload condition, as time becomes less crucial, other activities related to communication and flight progress exert greater positive and negative effects on the transitions to the next activity. The findings suggest that workload and stress affect time management in decision-making under the heavy workload condition, or in other words, time determines what is done.

After 10 years studying air traffic control, Sperandio $[24,25]$ noticed that the workload of the controllers increase with the traffic but this increase is regulated by changes in strategy. For a given situation and a given controller, certain procedures are more economic (i.e., require a smaller cognitive load) but as workload increases (i.e., the number of aircraft increases) the controllers spontaneously relax the performance criteria in order to adopt a qualitatively different strategy that accomplishes the task goals with less effort, in order to avoid crossing the overload threshold and delay disfunctioning [25]. At one particular airport it was observed that: 1) when there were one to three airplanes, the controllers nursed each airplane: they would calculate the optimal flight path for each individual airplane based on a number of variables such as speed, course, altitude and type of airplane; 2) when there were about four to six airplanes, they adopted a more economical strategy, such as adopting uniform speeds and stereotypical flight paths; 3) when there were more than six airplanes, the controllers would create waiting buffers that consisted of streams of planes, and brought them out of the buffer and toward the runway at a generally uniform speed on the same descent path. Because the load is so great, the controllers relax the performance criteria so that their only concern is safety rather than efficiency. The changes in strategy are not clear-cut and sudden, but there are statistical tendencies that vary according to contextual factors that are hard to anticipate. Also, the switch of strategy is dependent 
on the individual who might exhibit different tendencies on different days. Therefore, there is no magical number of aircraft that will always lead to a switch in strategy. Also, change of strategy is not a good indicator of workload [25]. These findings are in accordance to Woods et al. [31] who state that the cognitive and physical activities involved in the job of control vary between periods of low demand and periods of maximum occupation, when performance is more critical. In these situations, people adapt the activity continuously in order to respond to the variation in demand that will end up generating restrictions in cognitive activity, ranging from pressures and uncertainty to mistakes.

A study by Lamoureux [21] on the impact of aircraft proximity on the subjective mental workload of air traffic controllers showed that the number of aircraft controlled might not lead to high workload but what the aircraft is doing, especially in relation to other aircraft. This finding is consistent with a study by Falzon [11] who postulated that aircraft whose flight paths will not interact at any time in the near future are not considered by controllers to be contributing to their workload, but that aircraft whose flight paths will interact do contribute to subjective mental workload. Also, the manner in which the aircraft's flight paths will interact influences subjective mental workload. This leads to the conclusion that the underlying reason for a controller's mental workload has to do with the complexity of the relationships between aircraft (of which only a small part may be due to the number of aircraft in a sector), with the decisionmaking, mental calculations to ensure that separation is maintained, and projections involved in the successful air traffic control.

After revising the literature on workload of ATC controllers, Loft et al. [22] argue that the studies assume the ATC controller as a passive recipient of task demand. They do not explicitly take into account the fact that they can actively take steps that change task demand, so as to keep workload at an acceptable level. The authors believe that workload might be more strongly connected to the ATC controllers ability to manage their cognitive capacity, therefore, they propose taking a mental workload-centered approach rather than a task demand-centered one for understanding the ATC system.

The study presented in the next sessions aimed to approach the ATC as a cognitive system emphasizing the cognitive aspects involved in the different scenarios. According to Woods and Hollnagel [30], people adapt their tasks to respond to the variation in demands. Within this adaptation, basically, there are three classes of cognitive factors - knowledge, attention and strategy - which govern how people shape their intention for action: 1) knowledge factors encompass the mental model that the person has about a certain activity, how this knowledge is calibrated to perform the task, its level of precision and the extent to which analysis of the situation is simplified. Problems related to knowledge are basically exaggerated simplification, imperfection, contradiction, and incomplete mastery of knowledge, which may lead to failure or uncertainty in carrying out the task; 2) the dynamics of attention encompass perception of situations, and if the operator is fixed on specific points. Situational awareness is characterized by a variety of cognitive processing activities that are critical as to dynamism, to being event driven, and to the practice of multiple activities [30].

\section{Method}

The fieldwork took place between July and August 2006 and was anchored on six instruments: 1) direct observation; 2) filming (using three linked cameras) of the scenes under control and of the controllers carrying out the task; 3) semi-structured interviews; 4) discussion with the controllers about the characteristics of the cognitive factors (knowledge, strategy and attention dynamics); 5) questionnaire [26] for scene classification (degree of complexity / degree of risk) and prioritization of the cognitive factors involved; 6) adapted questionnaire [13] from the National Aeronautics and Space Administration task load index (NASA-TLX) [15] for workload evaluation.

Fourteen controllers and four coordinators of APPPA (with one year and six months to twenty-one years of experience) selected and evaluated the scenes (some only with the radar controllers or only with the coordinator controllers and others with both) giving a total of thirty-six interviews and evaluations of the degree of complexity/ risk and prioritization of the cognitive factors involved in the scenes. Seventeen APP-PA controllers responded the adapted NASA-TLX questionnaire, and seven took part in the general interview. All participants volunteered for the research and signed a consent form.

Based on the interviews, the real scenes' films and the questionnaires, the following were identified and analyzed: i) the perceptions of risk and complexity of the different air traffic scenes observed; ii) the prioritization of the cognitive factors involved in each task and; iii) the perception of the controller's workload. 
The statistical analysis was based on nonparametric hypothesis testing and multivariate technique of correspondence analysis. Mann-Whitney U test for two independent samples was used to verify the existence of significant difference between the function of the controllers at the APP-PA (radar or coordination), length of time in the profession, length of time at the APP-PA, in relation to the perception of risk and complexity of the scenes. Variance analysis by Kruskal-Wallis ANOVA test was used to verify the significant differences between the levels (Low, Medium, High) of both complexity and risk variables as well as the differences between the prioritization of the cognitive factors (Knowledge, Strategy and Attention dynamics) and the classification level of the scene (Easy, Medium or Difficult). Multivariate correspondence analysis was used to assess the correlation and intensity of association of the characteristics of the observed scenes with: the controller function at the APP-PA, their perception of risk/complexity and cognitive factors prioritization.

\section{Results}

\subsection{Characteristics of the scenarios}

Based on the film footage and field notebook, the scenes selected by the controllers display the following characteristics:

a) Number of Aircraft: this is the number of aircrafts involved during the length of the scene, including military aircrafts;

b) Military Aircraft: these are high-performance aircraft belonging to the Brazilian Air Force (FAB) generally used on military missions and which are involved during the length of the scene. They are known as Fighters, for they are used on search missions (hunting) and interception of other aircraft. Low-performance military aircraft such as aircraft for the transport of cargo or troops were excluded. The number military aircraft involved in the different scenarios observed varies from one to three. The aircraft fly both individually in different directions (to different sectors), as well as in formation, together, and in the same direction. Fighters appeared on eleven out of the total thirty-six observed scenes;

c) Procedures: some of the various following procedures appeared in some scenes. They have an impact on the conduct of the traffic, and therefore on the controller's decisions: (i) Precision Approach by Radar (PAR) is a precision approach conducted in ac- cordance with instructions issued by a radar controller, based on a precision approach radar display that shows the position of aircraft in terms of distance, azimuth and height. This equipment is different from the monitors with information from the radars normally used by controllers. In this procedure, the radar controller needs specifically to devote him/herself to guiding the aircraft to its final approach. It was undertaken whenever a military aircraft in the process of approaching Canoas aerodrome (the Canoas Air Force Base) requested this procedure as a form of training. The PAR procedure is performed on the console for normal operations, always the same one, by a controller other than those who were looking after the traffic. Of the thirty-six scenes, $14 \%$ use the PAR procedure; (ii) The Circling Approach is a complement of an approach procedure using instruments that requires the aircraft to perform, with visual references, a maneuver to circle the airfield and land. Many aircraft in training, and very often, low performance ones, carry out this procedure as training for landing and takeoff, and aborted landings. Of the thirty-six scenes, $8 \%$ contain the circling approach procedure; (iii) Visual approach is an in-flight approach by instruments, when part or all of the approach procedure by instruments is not completed and is undertaken with visual reference to the ground. Altogether, $78 \%$ of the scenes display aircraft performing the visual approach procedure. Cruising aircraft is when the aircraft cruise at the same flight level and maintain the minimum separation distances.

d) Meteorological conditions: these are the conditions expressed in terms of visibility, distance from the aircraft in relation to clouds and ceiling (cloud height). In the scenarios, the weather conditions are observed as: the presence of sun, clouds (cloudy, the worse situation being when there is a formation of cumulus nimbus clouds), rain. These weather conditions influence the flight conditions and may modify a procedure that hitherto was visual (visual flight), to flying by instruments. Another factor that incorporates weather conditions is wind speed and direction in the approach and take-off procedure. A tail-wind with a speed of around 5 knots can lead to an aircraft being unable to continue with the approach process, for it can be a limiting factor on the performance of the aircraft, or this may prevent it from taking off. It should also be mentioned that mist and fog leads to the aerodrome being closed for operations. Among the thirty-six scenes, thirty are in sunny conditions, three have the presence of clouds, and in three of them it is raining. In four scenes, the wind speed was 
close to the maximum limits for a safe approach by the aircraft (wind speed: high).

e) Runway in use: this is when the runway is being used for landing and takeoff. In the case of the scenes observed, this procedure refers only to the runway of the International Airport of Porto Alegre. At this airport, there is only one runway, but the letters $\mathrm{W}$ and $\mathrm{Z}$ refers to the direction of approach and takeoff: runway $\mathrm{W}$ indicates that the direction for landing and takeoff of aircraft is from west to east; runway $\mathrm{Z}$ indicates that the direction for landing and takeoff of aircraft is from east to west. Runway W has equipment that permits an ILS precision approach, the type of approach most commonly used and which provides greater margins of safety for aircraft. Runway $\mathrm{Z}$ does not have this. The choice of the runway to be used depends on the wind, as takeoff and landing are performed against the wind. This is why, most of the time, the average wind direction leads to runway $\mathrm{W}$ being used. Of the thirty-six scenes, $83 \%$ of them show runway $\mathrm{W}$ in use and $17 \%$ runway $\mathrm{Z}$ in use.

f) Radar service: this is the term used to describe a service provided directly by means of radar information. When observing the scenes, classification was made between radar control and non-radar control. Radar control is the term used to indicate that in the provision of the air traffic control service, information from the radar is being directly used. Non-radar control is when the information comes from a source other than radar, in this case, the pilot. Of the thirtysix scenes, $92 \%$ use radar control and $8 \%$ non-radar control.

\subsection{The impacts of the scenarios in the classification of the scenes}

The scenes show great variability as to their characteristics. The Easy scenes unfolded in good weather conditions, with runway $\mathrm{W}$ in use and radar control being used. $50 \%$ of the scenes are of Medium complexity and in them there is a greater presence of military aircraft. In one of these Medium scenes, there is non-radar control and there are different procedures to be performed. The Difficult scenes are when the weather was cloudy, military aircraft were cruising or these factors with a non-radar procedure with much traffic.

In the different scenarios observed, factors related to the weather (wind speed, sunny, clouds and rain) are the ones most subject to variation and, when there are unexpected changes, this can directly influence the safety of managing the traffic. When combined with a greater number of procedures, they further increase the complexity of the scene. Cloud is a fundamental factor because it changes the expected behavior, namely it alters the controller's prior planning, thus increasing the level of complexity of the scene since the factor of surprise increases the need for improvisation. When in the route of the aircraft there are large cloud formations, the aircraft tend to take a detour, because cloud can lead to turbulence (and discomfort for passengers). Added to this, a large number of aircraft tend to converge to the same point.

The technology factor (in the case of non-radar control) may influence the complexity of the scene, since communication occurs only via radio frequency without radar visualization. Another problem is the presence of military aircraft flying in the sector, because their high performance impacts on the time the controller takes to make a decision. These cases show the need for the controller to review future situations of the scenario in order to predict the potential conflicts [10].

\subsection{Statistical analysis of the results}

Mann-Whitney $U$ test shows that there is no significant difference either between the function of the controllers (radar or coordination) at the APP-PA (U (diff) $=-0.049, \mathrm{p}=0.961$ ) or the length of time in the profession and in APP-PA $(\mathrm{p}=0.248$ and $\mathrm{p}=0.363$, respectively) as to the perceived degree of complexity and risk of the scene. However, Kruskal-Wallis ANOVA shows that there are significant differences for the perceived degree of complexity that leads to the classification of a scene either as Easy, Medium or Difficult $($ Chi $(5.991)=10.5, p<0.01)$, i.e., the perception of complexity and the classification of the scene followed the same behavior, the greater the complexity, the higher the classification the scene. As to the degree of risk $(\mathrm{Chi}(5.991)=12.0, \mathrm{p}<0.01)$, the classifications into Easy and Medium scenes are similar, and these differ significantly from the Difficult scene. Only few Difficult scenes were considered under risk of a mishap, making it clear that the controllers understand that a risky situation is one in which an incident might occur.

Kruskal-Wallis ANOVA showed a significant difference among the levels of the scene classification $($ Chi $(5.991)=6.52, p=0.034)$. The Easy and Medium levels showed to be similar and significantly differing from the Difficult level. There is no correlation between the variable type of controller and the level of scene classification For both controllers, the 
scenes are Easy when there is little traffic, which is associated with the lack of possible conflicts, and the situations are easily resolved, based on the rules. Medium scenes present conflicts, aircraft of different performances, including military ones. In Difficult scenes, there is more aircraft crossing, traffic from different sectors and military aircraft, non-radar operation and unfavorable meteorological conditions.

There is a linear correlation between the level of scene classification and the variables "meteorological conditions" and "number of aircraft". From this analysis, an attempt was made to assess the level of association between the variables that showed a significant linear correlation. The Easy scene is significantly associated with the level of "sunlight" while the Difficult scene is significantly associated with the level of "cloud". The Easy scene is significantly associated with "four" aircraft while the medium scene is significantly associated with "six" aircraft under control. The Difficult level is associated with "seven or more" aircraft what is in accordance with Coeterier [7], Kallus et al. [19], Sperandio [24,25] but in disagreement with Lamoreaux [21] and Falzon [11]. It should be noted that controlling more than seven aircraft is considered difficult, although it is less than the maximum permitted number of ten aircraft laid down by aeronautical legislation ICA 100-30 [4] for APP-PA. Correspondence Analysis showed that both the coordinator and the radar controllers consider a scene is Easy when it is "sunny" and there are "three" or "four" aircraft under control. The radar and coordinator controllers consider a scene is Difficult when there are more than "seven" aircraft under control and the weather is "cloudy". The radar controller considers a scene is Medium when there are "five" or "six" aircraft under control.

Kruskal-Wallis ANOVA showed a significant difference among cognitive factors (Attention, Strategy and Knowledge) prioritization (Chi $(5.991)=22.0, p$ $<0.01$ ), the radar and coordinator controllers prioritizing differently depending on the complexity of the scene. For the radar controller, Strategy is the most important factor in both Easy and Medium scenes while the coordinator controller prioritizes Knowledge in an Easy scene and Attention in a Medium one. In a Difficult scene, both the coordinator and radar controllers consider that Attention is the most important factor. Strategy, for the controllers is related to actions need to restrict the flight level of an aircraft and resolve the situations. Knowledge is linked to the rules, and, especially, isolated concepts such as, the performance of the aircraft. The fact of having experienced a similar situation with aircraft of different performance was singled out as being very important.

The adapted NASA-TLX results showed that mental load demand, mainly because of the weather conditions and phraseology, has the greater impact on the activity of the controllers. The level of pressure imposed in order to undertake the time demand came second, what is expected since APP controllers need to guide traffic in such a way that this does not create delays and dissatisfaction, both among colleagues (airport Tower, ACC and/or COPM controllers) and the pilots of the aircraft. The physical load is what has least impact on the activity.

On April 2007, the research as discussed in a meeting with the APP-PA controllers (whether or not they had taken part in the research), when many results could be elucidated and explained.

\section{Conclusions}

This study on the activities of the air traffic controllers of the APP-PA intended to contribute to the understanding of the work performed at the Brazilian ATC system that is operating in the limit of its capacity and therefore, in the edge of safety operation. Weather conditions (i.e. cloudy) and number of aircraft (seven or more) under control were the most important contributing variables for the complexity of the task. Mental and time demands have the greatest impact on workload. However, the interviews showed that controllers work under pressure due to the reduced number of controllers, the lack of training, autonomy and teamwork. These problems are difficult to solve in the present Brazilian scenario, since controllers do not stay long in their jobs, and they are under military coordination that makes the decisions about the teams and shiftwork. If no teams are ever build, coordination, integration and knowledge sharing among controllers is not possible. There is an urgent need for hiring and training more controllers, who need more autonomy to engage with the colleagues and decide under serious situations.

It is important to note that this study was only possible due to the interest of the Department of Airspace Control (DECEA) and the participation and interest of the controllers and senior managers in the research. For the latters, the study supplies suggestions for training controllers who enter APP-PA. For the controllers, the authorization for developing a research in an environment of rigid rules, and, very often, one of difficult access, the study was seen as a 
valorization of their work, specially if it is considered that it was carried out at a time of serious crisis for the Brazilian air sector. It is worth emphasizing that, despite the crisis, the field study was not affected by the air traffic control strike, which occurred in other regions of Brazil. Although the climate of uncertainties and tensions was present in the final two months of the research, the controllers, senior managers and DECEA never stop supporting the study.

\section{Acknolewdgements}

The authors are grateful to DECEA for the authorization, and to all controllers and senior managers engaged in this research, which was partly funded by the Brazilian Federal Agency for the Support and Evaluation of Graduate Education (CAPES).

\section{References}

[1] Airsafetygroup. O Controle de tráfego aéreo e a prevenção de acidentesaeronáuticos,2005.http:// www.airsafetygroup.com.br Accessed May 5, 2005.

[2] S. Banbury and S. Tremblay, A cognitive approach to situation awareness: theory and application, in: Team situation awareness as communicative practices, C Garbis and H. Artman, ed., Hampshire: Ashgate, 2004.

[3] C. Boag, A. Neal, S. Loft and G. S. Halford, An analysis of relational complexity in an air traffic control conflict detection task, Ergonomics 49 (14) (2006), 1508-1526.

[4] Brasil, Ministério da Defesa, Comando da Aeronáutica, Tráfego Aéreo, ICA 100-30: Planejamento de pessoal ATC. Brasília: Comando da Aeronáutica, 2008, p 42.

[5] Y-H Chang and C-H Yeh, Human performance interfaces in air traffic control, Applied Ergonomics 41 (1) (2010), 123-129.

[6] Civil Aviation Authority, CAP 413: Radiotelephony Manual, ed. 15, Gatwick, UK, Safety Regulation Group, Civil Aviation Authority, 2004.

[7] J.F. Coeterier, Individual strategies in ATC freedom and choice, Ergonomics 14 (5) (1971), 579-584.

[8] G. Costa, Evaluation of workload in air traffic controllers, Ergonomics 36 (9) (1993), $1111-1120$.

[9] M. B. Edwards, D. K. Fuller, O. U. Vortac and C. A. Manning, The role of flight progress strips in en route air traffic control: a time-series analysis, International Journal of Human - Computer Studies 43 (1) (1995), 1 - 13.

[10] K. Eyferth, C. Niessen and O. Spaeth, A Model of Air Traffic Controllers' Conflict Detection and Conflict Resolution, Aerospace Science and Technology 7 (6) (2003), 409-416.

[11]P. Falzon, Display structures: compatibility with the operator's mental representation and reasoning process, in: 2nd European Annual Conference on Human Decision Making and Manual Control, Delft, North Holland Publishing Co., 1982, 297 - 305.

[12] J. G. N. Farias, Choque de Gestão: do vôo 1907 ao apagão aéreo no Brasil, Rio de Janeiro, Brazil, Ciência Moderna, 2007.

[13]L. B. M Guimarães and R. L. Diniz, Adaptation of the NASATLX questionnaire, 2001. First used in: R.L. Diniz, Avaliação das Demandas Física e Mental no Trabalho do Cirurgião em Procedimentos Eletivos, Thesis (Dr.) Federal University of Rio Grande do Sul, Porto Alegre, 2003.
[14]G.A. Hadley, J.A. Guttman and P.G. Stinger, Air traffic specialist performance measurement data base, Federal Aviation Administration DOT/FAA/CT-TN99/ 17, Oklahoma City, FAA, 1999.

[15] S.G. Hart and L.E. Staveland, NASA Task Load Index (TLX): Computerized Version. (1986), Moffett Field, CA: NASAAmes Research Center, Aerospace Human Factors Research Division, 1988.

[16]E., Hollnagel, Cognition as control: a pragmatic approach to the modelling of joint cognitive systems. Department of Computer and Information Science University of Linkönping, Linkönping, Sweden, 2002.

[17] International Organization for Standardization ISO 100752:1996, Ergonomic principles related to mental workload -Part 2: Design principles, 1996, 1-11.

[18] International Organization for Standardization ISO 10075 3:2004, Ergonomic principles related to mental workload -Part 3: Principles and requirements concerning methods for measuring and assessing mental workload, 2004, 1-15.

[19]K.W Kallus, P. Hoffmann, H. Winkler and E. M. Vormayr, The taskload-efficiency-safety-buffer triangle - development and validation with air traffic management, Ergonomics 53 (2) (2010), 240-246.

[20] G. Kuk, M. Arnold and F. E. Ritter, Effects of light and heavy workload on air traffic tactical operations: a hazard rate model, Ergonomics 42 (9) (1999), $1133-1148$.

[21]T. Lamoureux, The influence of aircraft proximity data on the subjective mental workload of controllers on the air traffic control task, Ergonomics 42 (1999), 1482-1491.

[22] S. Loft, P. Sanderson, A. Neal and M. Mooij, Modeling and predicting mental workload in en route air traffic control: critical review and broader implications, Human Factors 49 (3) (2007), 376-399.

[23] Ch.H. Morris and Y.K. Leung, Pilot mental workload: how well do pilots really perform?, Ergonomics 49 (15) (2006),1581-1596.

[24]J-C. Sperandio, Variation of Operator's Strategies and regulating effects on workload, Ergonomics 14 (5) (1971), 571-577.

[25]J-C. Sperandio, The regulation of working methods as a function of work-load among air-traffic controllers, Ergonomics 21 (2) (1978), 195-202.

[26]C.V. Vargas, Análise ergonômica do trabalho de controle de aproximação aérea (APP) em situação real, Dissertation (MEng.), Federal University of Rio Grande do Sul, Porto Alegre, 2007.

[27] Veja Revista, Caos Aéreo, 2007, http://veja.abril.com.br/idade/exclusivo/perguntas respostas/ca os aereo/index.shtml. Accessed on March 23, 2009.

[28]J. Vogt, L. Adolph, T Ayan, A Udovic and M Kastner, Stress in modern air traffic control systems and potential influence on memory. Human Factors and Aerospace Safety Vol 2 (4) (2002), 355-378.

[29] O. U. Vortac, M. B. Edwargsand and C. A. Manning, Sequences of actions for individual and team of air traffic controllers, Human-Computer Interaction 9 (3-4) (1994), 319- 343

[30]D.D. Woods and E. Hollnagel, Joint cognitive systems: patterns in cognitive systems engineering, Boca Raton, CRC/Taylor \& Francis, 2006.

[31]D.D. Woods, L. J. Johannesen, R. I. Cook and N. B. Sarter, Behind human error: cognitive systems, computers, and hindsight, Columbus, Ohio, CSERIAC, 1994.

[32]H. Zeier, Workload and psychophysiological stress reactions in air traffic controllers, Ergonomics 37 (3) (1994), 525 -539. 